\title{
AC 2007-658: FRESHMAN PROJECT: AUTONOMOUS UNDERWATER VEHICLE (AUV)
}

\section{David Ye, Polytechnic University}

David Ye is a senior head teaching assistant in General Engineering. He expects to receive his BSEE from Polytechnic University in June 2007. His interests include robotics. He interned at Symbol Technologies researching wireless protocols and Power LEDs.

\section{Ilya Brutman, Polytechnic University}

Ilya Brutman is a teaching assistant in General Engineering. He expects to receive his BSCompE from Polytechnic University in June 2008.

\section{Gunter Georgi, Polytechnic University}

Gunter W. Georgi is an Industry Professor at Polytechnic University. He received his BS from Cooper Union and his MS and professional M. E. Degrees from Columbia University. He is a registered Professional Engineer. He has worked for many years in the aerospace industry in design, analysis and management functions, including Thermal Mission Analysis of the Lunar Module from Apollo Project.

\section{Lorcan Folan, Polytechnic University}

Lorcan M. Folan is Head of the Department of Physics at Polytechnic University. He holds a B. Sc in Applied Science from Trinity College, Dublin and M.S. and Ph. D. degrees in Physics from Polytechnic University. 


\title{
Freshman Project: Autonomous Underwater Vehicle (AUV)
}

\author{
David Ye, Ilya Brutman, Gunter W. Georgi , and Lorcan M. Folan \\ Polytechnic University
}

\begin{abstract}
Like many other schools, our school has developed robotics projects using commercial products such as Lego Mindstorms ${ }^{\circledR}$ for its Freshman Engineering course. These projects have been wellreceived by many students ${ }^{1}$. However, some Freshman students have commented that they wanted something different and more challenging than land-based robotics.

To meet this demand, we have developed an underwater test field that requires an autonomous underwater vehicle (AUV) to perform various tasks. These tasks necessitate the design and construction of AUVs that operate on the surface of water, on the bottom surface under water, as well as when running submerged in between. The construction cost for material for the underwater test field was kept below $\$ 1000$.

Many students are familiar with LEGO robotics on dry land. AUVs present many new challenges: waterproofing conventional LEGO components, buoyancy, balance, and 3-D motion. Since completion of various tasks contributes different point values, the final total point value is a measure of how well the AUV has performed. This is the basis for a lively design competition between Freshman Engineering teams.
\end{abstract}

\section{Introduction}

Polytechnic University's Introduction to Engineering and Design course consists of lectures (1 $\mathrm{hr} / \mathrm{wk}$ ), laboratory work ( $3 \mathrm{hrs} / \mathrm{wk}$ ), and recitations ( $2 \mathrm{hrs} / \mathrm{wk}$ ) for an academic semester. Engineering activities and design examples from a variety of engineering disciplines are presented and a selection of professional tools (MS Word, MS Excel, MS PowerPoint, MS Project, AutoCAD, LabView) are introduced. Students are exposed to team building activities and must make presentations (both written and oral), as an individual and as a member of a team. Some laboratory work involves design competitions and the students must select one of several semester-long design projects that require teamwork and develop project management skills.

For the Fall of 2006 for the first time ten student teams of 3 students each designed AUVs. All teams completed successfully. Of the ten designs, 7 were bottom travelers, 1 was a surface vessel, and 2 were floaters. All teams were very enthusiastic and put in many hours to solve engineering problems and unexpected challenges posed by buoyancy, water resistance, and orientation/location problems.

The first priority was to provide a working environment for the AUVs; a complete assessment of the project still has to be done. Below are two examples of AUVs built by the students during the Fall 2006 semester. 


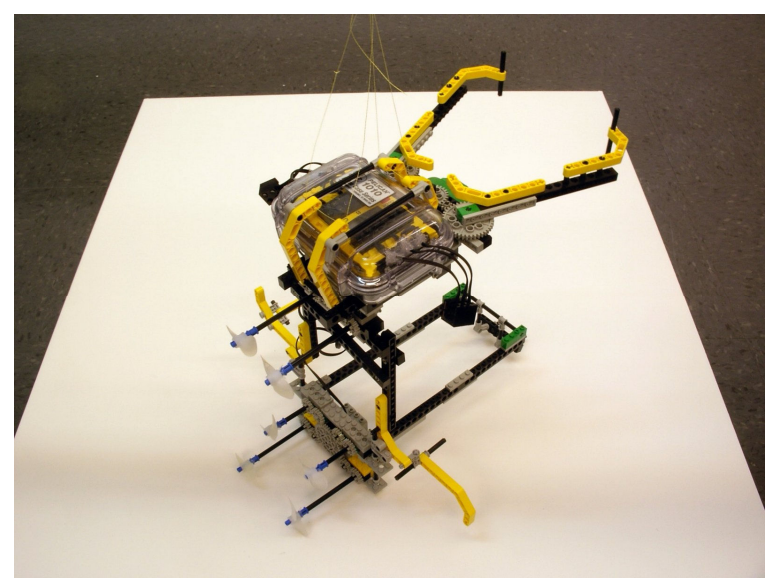

Figure 1 Completed AUV

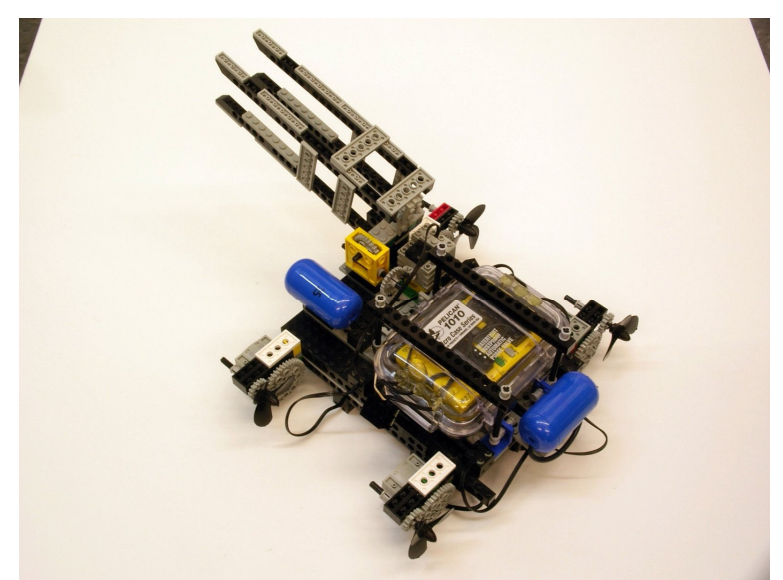

Figure 2 Completed AUV

\section{AUV Design Project Problem Statement}

After deciding that an AUV design project would be offered to freshmen students in the current Introduction to Engineering Course ${ }^{2}$, three problems had to be resolved:

1. Build an inexpensive and small underwater test field that would allow the students' AUVs to be tested as well as permit easy modification of the terrain seen by the AUV.

2. Decide on tasks that the AUVs should perform and assign achievement points for completion of the tasks.

3. Provide basic underwater components to the students so that they could achieve their tasks without doing major research on underwater functionality.

In addition, assessment would have to be made to determine whether the new AUV design project was favorably received by the students and if the greater complexity of the project compared to other offered design projects was of benefit.

\section{AUV Design Challenge}

In the early part of the semester, students select a project they would like to work on and teams are formed. There are six projects for them to choose from: four robotic projects ( 3 land based, one water-based: the AUV), a train digital logic design system ${ }^{3}$, and a supermarket design. The deadline for each project is on the last day of class. Along with the final project deadline there are also several milestones that the student teams must meet throughout the course of the semester.

\subsection{AUV Request for proposal}


The student teams must answer a Request for Proposal (RFP) for the AUV. Specific tasks are delineated as well as the credit points they get for completing specific tasks.

Specific rules:

- The AUV must be able to navigate autonomously around the test tank. The robot must achieve at least 100 points in 5 minutes by picking up or moving various objects.

- Objects include "data canisters" (ping pong balls), "plants" (Lego rings), seismic instrument. See below:
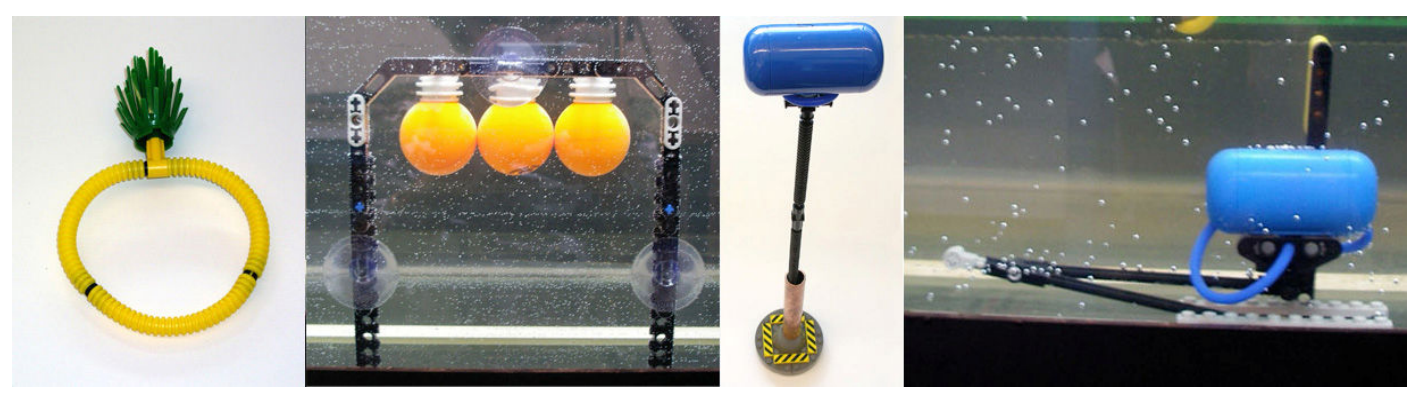

Figure 3 Field objects

- The finished AUV must fit inside a 1 foot by 1 foot by 1 foot box.

- The AUV must start in the starting area (the left third of the tank). The AUV can start at any depth, facing any direction. The AUV may not be touching any of the field objects during the initial start.

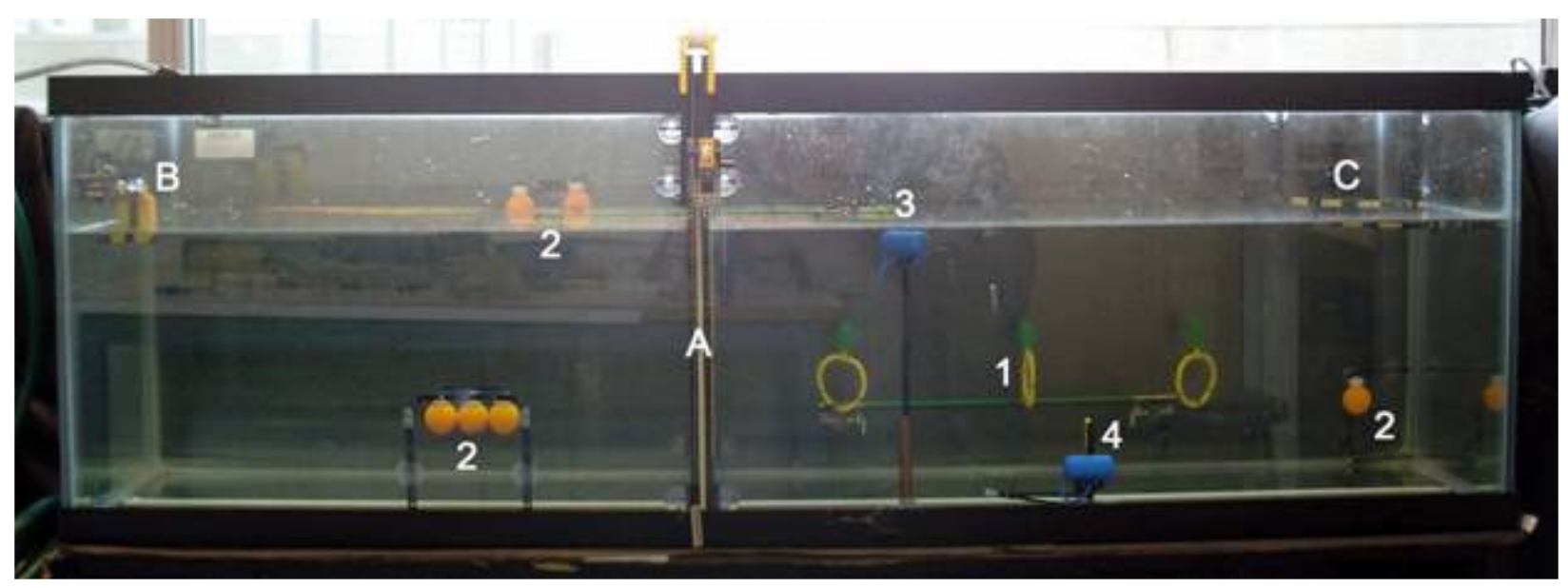

Figure 4 The test tank

\subsection{Design Challenges for Students}

Designing an underwater vehicle presents many new challenges that are not met when creating a land-based robot. The robot must now be able to navigate in a three-dimensional field. Not only are there target objects in front, back, left and right of the robot, they are now also on top and on the bottom. 
The way the robot maneuvers around the test tank is also different. A traditional robot would use wheels, tread or perhaps legs to move around. This is also true if a robot is designed to work on the ground at the bottom of the tank. However, in addition the robot can use propellers and flaps underwater.

Students' AUV designs can usually be classified as one of three different classes: a skimmer (top of water surface), a rover (bottom surface) or a hoverer (float between top and bottom surfaces). Each class of AUV has its advantage over the others. An AUV that only drives on the bottom has an easier time navigating but a hard time reaching objects on the surface level. An AUV that only floats on the surface of the water doesn't have as many obstacles in its way but has a harder time reaching the objects on the bottom. An AUV that is able to move up and down in the water has an easier time reaching all the objects but the AUV itself is more difficult to design in the first place.

\subsection{Examples of finished AUVs}

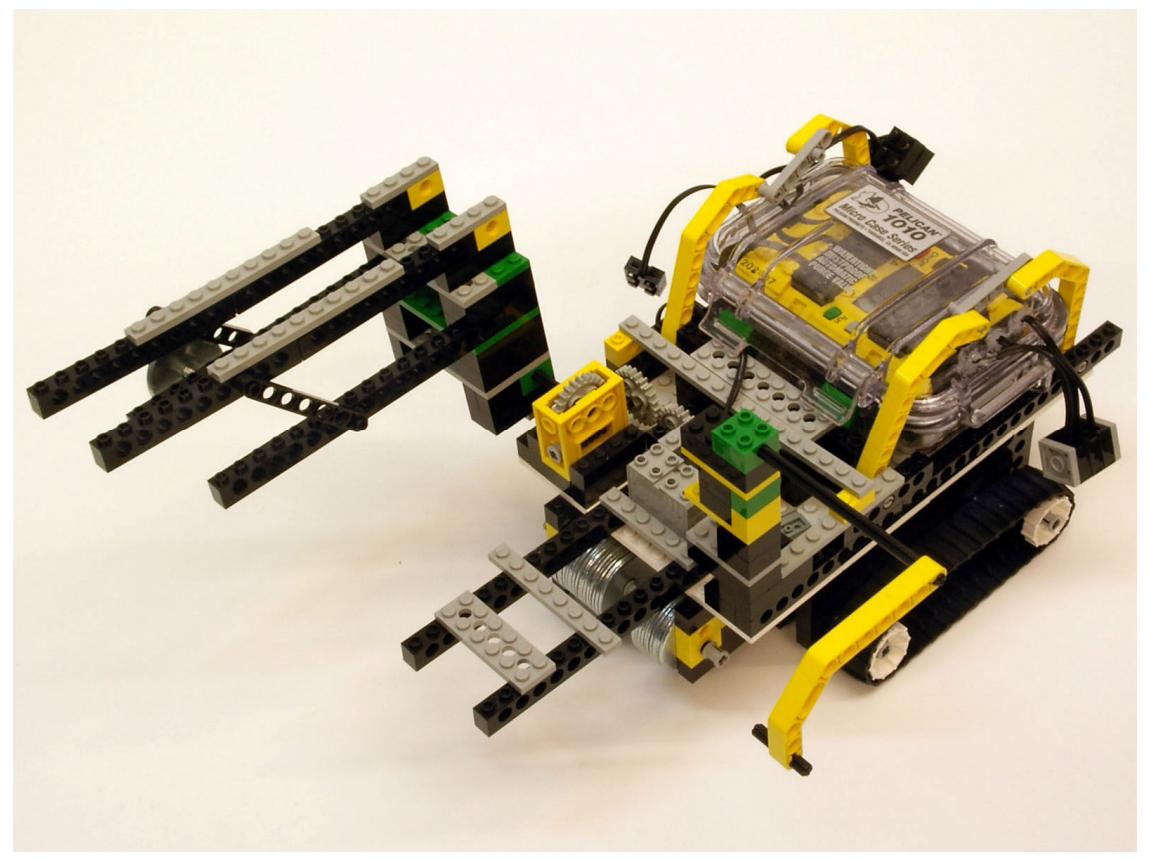

\section{Figure 5}

A bottom surface rover. Uses its front claw to collect ping pong balls. The extra weight near the bottom ensures that the treads have enough traction to move even with the cargo of ping pong balls. 

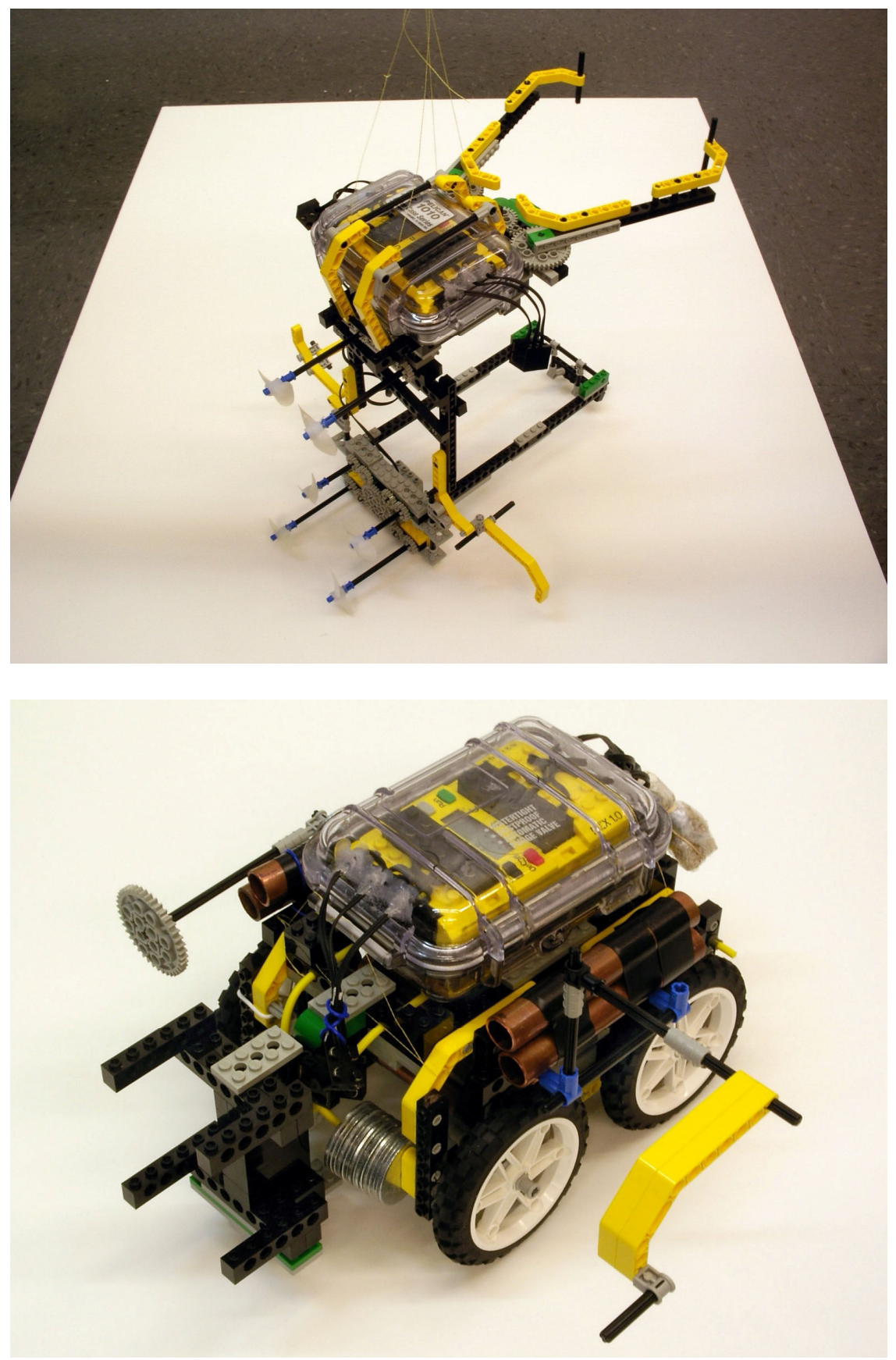

\section{Figure 6}

A surface skimmer. Uses its claw to grasp ping pong balls on the surface of the water. The bottom part is used for moving objects underwater. Propellers are used for propulsion.

\section{Figure 7}

A bottom surface rover. Uses a hook to collect underwater Lego rings and a front grill to push objects. Extra weight near the wheels provides additional traction and control. 


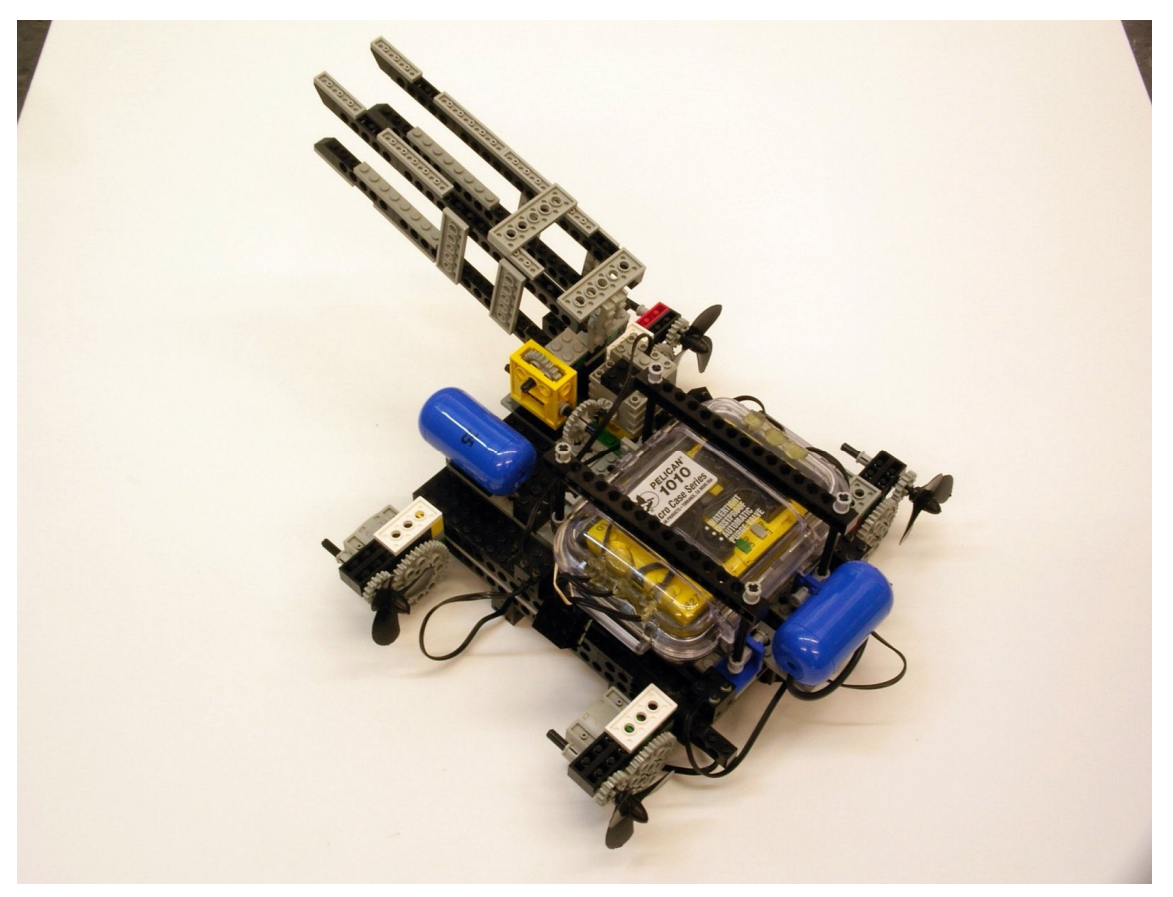

\section{Figure 8}

A hoverer that is able to float at any depth. Its four propellers tilt up and down allowing it to move vertically as well as horizontally in the water. It uses its front claw to scoop up ping pong balls.

\section{AUV Design Development}

To use a robot under water, the major robot components all had to be modified for under-water use: the control unit (RCX), the sensors, and the mode of propulsion.

\subsection{Control Unit}

To achieve full autonomy the underwater robots must be controlled by an onboard microcontroller. It was decided to use the Lego RCX for this role because the RCX requires little modification in order to have a fully operational robot. Enough RCXs already existed in our inventory; and using RCXs gave the following benefits:

- The RCX uses an infrared port for communication. Programs are sent to the RCX without plugging any wires into it. This allows uploading programs to it without having to open its water proof housing. It also allows controlling the RCX while it is submerged in the water using the standard Lego remote control. With the remote it is easy to start or stop a program stored on the RCX or control the motors connected to it manually.

- The RCX is programmed with Robolab. Robolab is a graphical based programming language similar to LabView. It is easy to learn and has real world application.

- The RCX allows using existing Lego sensors with the new underwater robots. This eliminates the cost of having to obtain new sensors; the sensors that where designed for the RCX can be used with minimal modifications.

The RCX also has disadvantages. Mainly: 
- The RCX doesn't work underwater. The RCX, like most electrical devices, was designed to work on dry land. To get it to function underwater it must be placed inside a waterproof case.

- The water-proof case which encompasses the RCX is huge (compared to the rest of the Lego). As snug as the RCX fits inside the case, it is still the biggest component, and makes up for about half the size of the AUV most of the time.

- The sensors used by the RCX are designed to work on dry land and must be water proofed before they can be used underwater.

The Pelican 1010 case was chosen for the RCX's water-proof housing. To be able to connect motors and sensor to the RCX while inside the case, six holes were drilled into the Pelican case: three holes for the input wires and three for the output wires. The wires were then inserted into the appropriate holes. Marine GOOP was applied to the holes to form a water tight seal around the wires. To get a Lego wire to fit into the small holes, one connector head of the wire was removed. The wire was then inserted into the case and the Lego connector head re-attached to the wire.

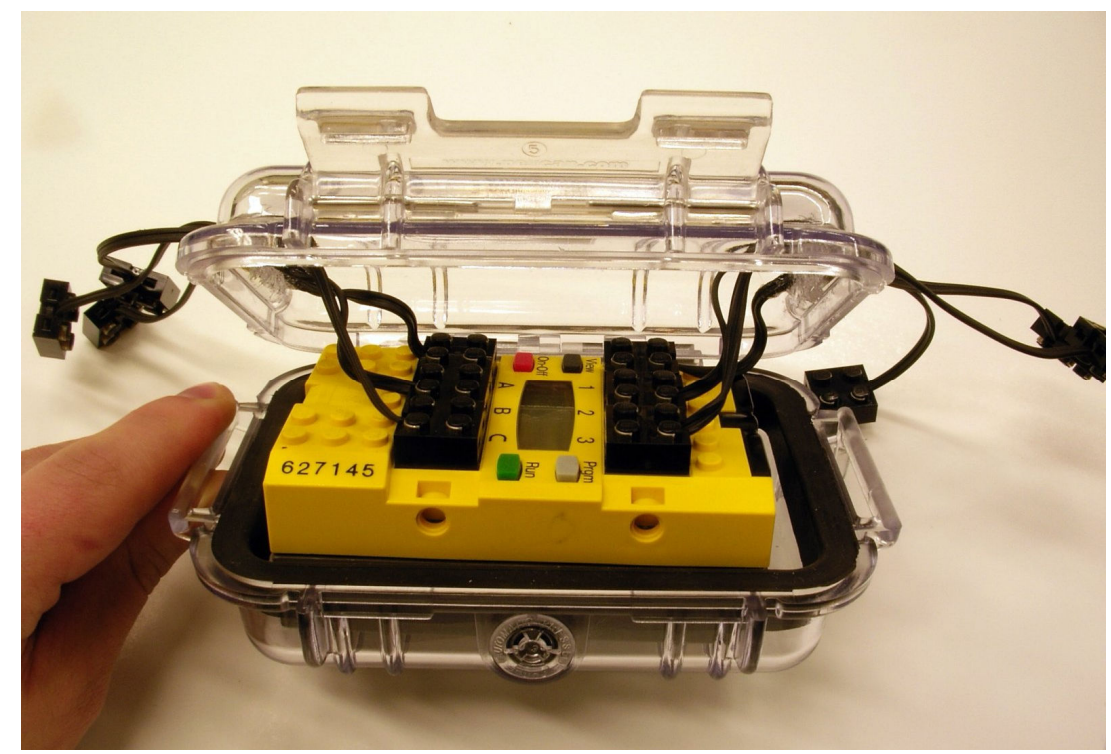

Figure 9 RCX inside a modified Pelican 1010 case

\subsection{Sensors}

The Lego touch sensor is the easiest of the sensors to work with underwater. It works as well underwater as it does on dry land. The interference of the water has no effect on it. It can be attached at an end of a Lego wire and expected to work reliably. The only time when the touch sensor might give false readings is when the water gets too conductive. In this case the touch sensor's reading is a false positive. This problem is prevented if the water is kept clean all the time. 
The Lego light sensor has a circuit inside and needs to be water-proofed before it will work underwater. To make the light sensor water-proof a hole was carefully drilled in the bottom of the sensor housing. Epoxy was then injected until the circuit board inside was fully covered. Once the epoxy dries the sensor is able to function underwater just as well as it does on dry land. The only side effect of the water-proofing is that the range of values that the light sensor can sense decreases by about five units from each side of the normal range (1-100).

All Lego wire connections leading to a light sensor must also be water-proofed. The RCX measures the value from the light sensor by measuring the resistance across it. An inconsistency in measurement occurs when water touches the exposed Lego connector at the junction of two wires. The water between the two Lego terminals adds an additional resistance in parallel to the light sensor. This added resistance might be stable but it doesn't matter since it is low enough to saturate the readings from the light sensor resulting in a constant reading of ' 100 '. A successful method of water proofing the wire connections is to wrap it in plastic wrap. Adhesive putty can also be used to form a better seal around the wires and the plastic wrap. This method is both reliable and temporary. The sensors connected to the wires can be swapped as many times as needed.

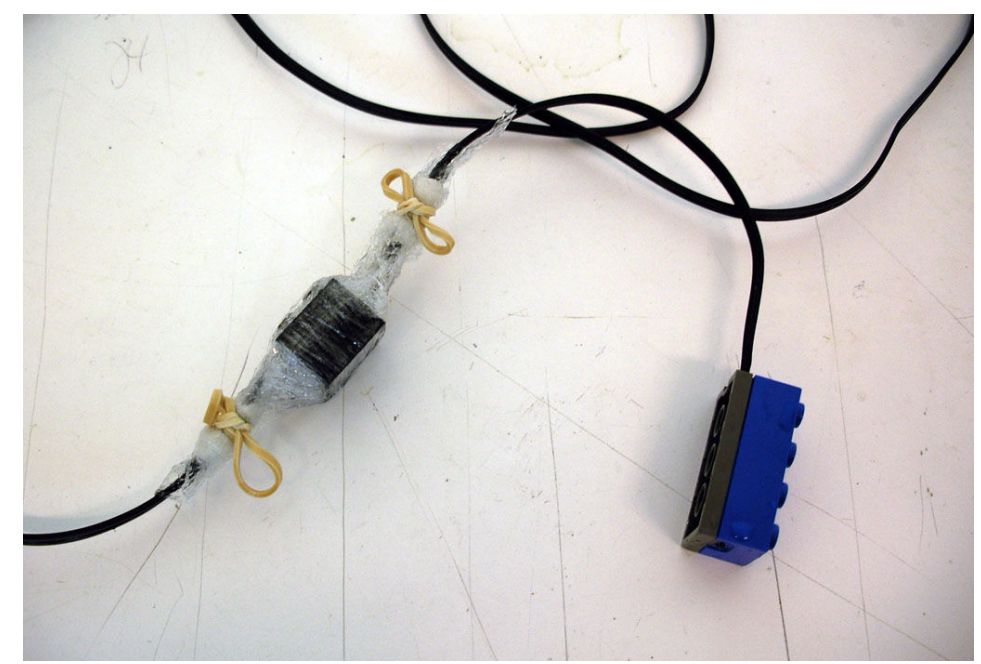

Figure 10 Water-proofed wire junction and light sensor

The inconvenience of having to water-proof every wire connection has lead to the discovery of a new type of sensor. It is possible to detect the water level with just the use of a single Lego wire. In Robolab ${ }^{\circledR}$, you can treat the plain Lego wire connected to an input port of the RCX as a light sensor. When the free (unconnected) end of the Lego wire is submerged into the water, the RCX will get a 'light intensity' reading of greater than 80 . When the free end of the wire is taken out of the water, the RCX will read a value of about 10. Students can use this technique to allow their AUV to know when it has surfaced and when it is submerged. 


\subsection{Programming}

Lego RoboLab was used for programming; it is a graphical icon based programming language similar to LabVIEW. It is an easy language to learn and allows students to access advanced features of the RCX microcontroller without the need to learn advanced syntax.

\subsection{Propulsion}

Students were provided with Lego compatible propellers. The propellers are made simply by inserting a Lego piece covered in plastic wrap into the hole of the propeller. The plastic wrap between the propeller and the Lego shaft ensures that the propeller stays on snug and doesn't fall off. Lego DC motors are used on the AUVs to provide motion. They have no trouble running underwater.

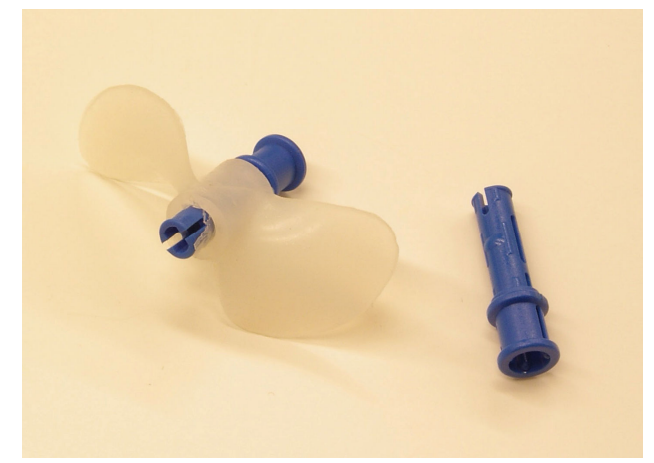

\section{Figure 11 Propeller attached to a Lego piece}

\subsection{Testing Area}

The 125 gallon aquarium that houses the AUV course sits inside a spill basin. The basin is designed to catch the water if the aquarium ever leaks or breaks. The basin is about 7 feet wide and 8 feet long. Its structure was constructed out of wooden 2-by-4's and covered by two layers of 45 mil thick pond liner. If the aquarium ever breaks and the basin floods with water, a sump pump will be used to pump the water out of the basin and into the sink located next to the aquarium. There is a 1 inch layer of dense Styrofoam underneath the pond liner inside the basin. The Styrofoam is placed from the end of the basin up to the legs of the aquarium stand. If the basin ever floods, the water will flow into a 1 inch deep ditch below the aquarium, from where the sump pump will have an easy time pumping it out. The same sump pump is also used to pump the water from the aquarium into the nearby sink, and to fill the aquarium with the water from the sink. 


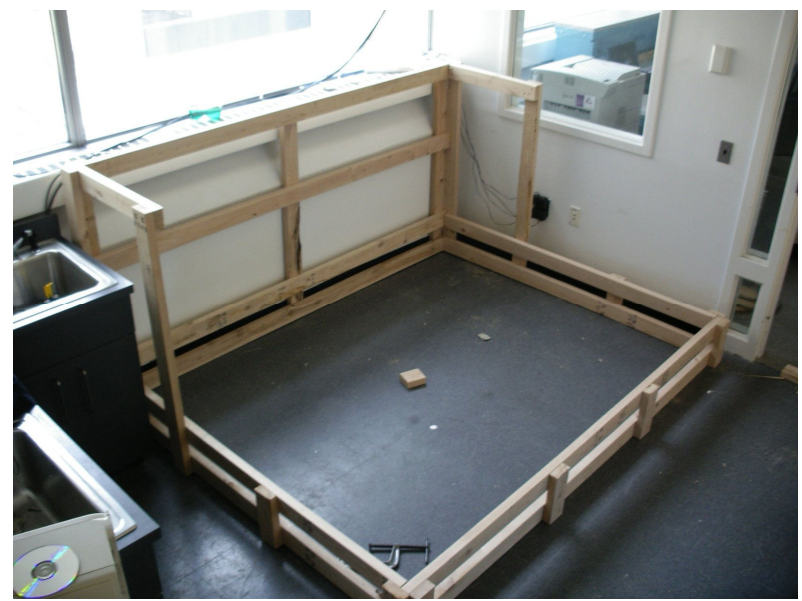

Figure 12 Basin under construction

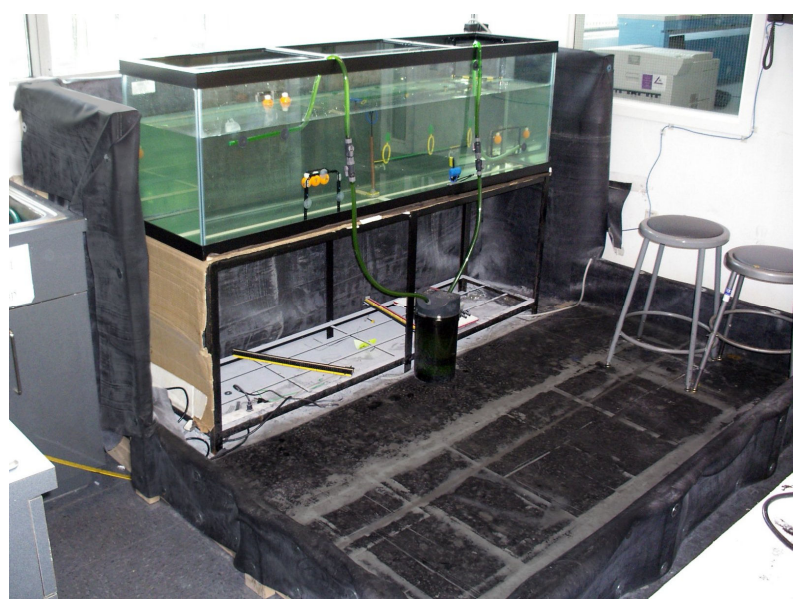

Figure 13 Aquarium inside the basin

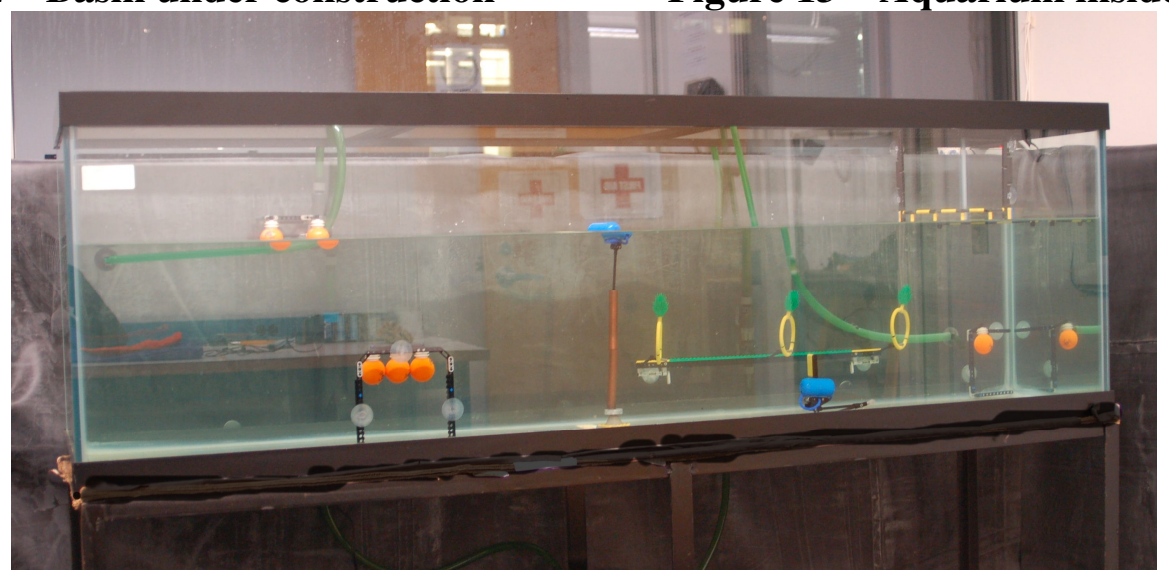

Figure 14 Aquarium inside the basin

The field elements inside the aquarium are made entirely out of Lego components. They are attached securely to the glass surface with suction cups. The suction cups have a hole on their back side into which Lego axles fit perfectly.

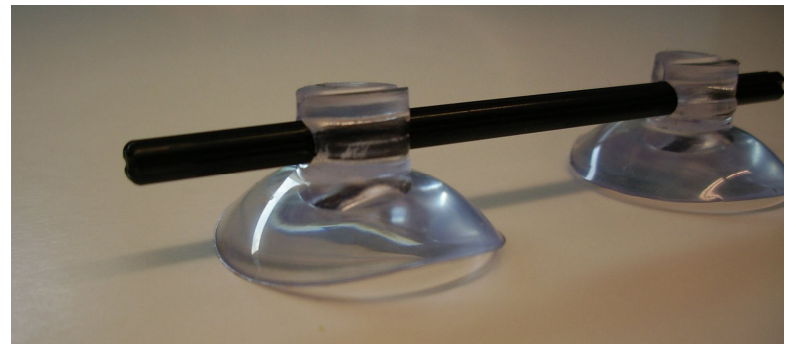

Figure 15 Suction cups attached to a Lego axle

A canister filter is used to keep the water inside the aquarium clean. The filter's hoses are removed from the aquarium when the aquarium is in use, so as not to get in the way of the student's AUVs. The filter cleans the water when the aquarium is not in use, mostly during the mornings, nights, and weekends. 


\subsection{Cost}

The total cost of the underwater test field was $\$ 819$. A summary of the most expensive items purchased is given in the table below.

\begin{tabular}{|l|r|c|c|}
\hline \hline Item & Price & Quantity & Total \\
\hline All Glass 125 Gallon Aquarium & $\$ 234$ & 1 & $\$ 234$ \\
\hline Canister Filter & $\$ 120$ & 1 & $\$ 120$ \\
\hline Pond Liner (45 Mil EPDM Firestone 10'x15') & $\$ 87$ & 1 & $\$ 87$ \\
\hline Aquarium Stand & $\$ 80$ & 1 & $\$ 80$ \\
\hline Pond Liner (45 Mil EPDM Firestone 10'x10') & $\$ 58$ & 1 & $\$ 58$ \\
\hline Lumber, misc. hardware & $\$ 298$ & 1 & $\$ 298$ \\
\hline TOTAL & & & $\$ 819$ \\
\hline \hline
\end{tabular}

Initially ten AUV kits were built with a cost of $\$ 212$ per kit. The course already had an abundance of Mindstorms kits so modifying them only required the expenditure of waterproofing supplies.

\begin{tabular}{|l|r|c|c|}
\hline Item & Price & Quantity & Total \\
\hline Lego Mindstorms Kit & $\$ 200$ & 1 & $\$ 200$ \\
\hline Pelican Case & $\$ 7$ & 1 & $\$ 7$ \\
\hline Propellers & $\$ 3$ & 1 & $\$ 3$ \\
\hline Waterproofing Supplies & $\$ 2$ & 1 & $\$ 2$ \\
\hline TOTAL & & & $\$ 212$ \\
\hline \hline
\end{tabular}

\section{Future}

The idea of using $3^{\text {rd }}$ party sensors along with the Lego sensors has held our interest ever since the beginning of this project. It is easy to see the benefits a compass sensor or a water pressure sensor will have for an AUV. A RCX compatible compass sensor sells for around $\$ 80$. 
A less expensive method is using reed switches to detect the presence of a strong magnetic field. Small magnets could be attached to field elements and a 'magnet sensor' on the AUV would be able to sense it.

Our ongoing work includes researching the possibility of integrating the recent release of Lego Mindstorms NXT into the AUV project. The NXT has new challenges for working underwater. The new NXT brick is bigger than the previous RCX. The new motors can't be used directly underwater since they are now digital servos and would be affected by water. The new NXT also open up the possibility of using Bluetooth on the AUVs.

\section{Assessment}

The first semester of the project was well received by students, many felt it was more exciting than the other projects offered by the course. Many of the students underestimated the difficulties they would encounter when working in a 3 dimensional environment. Most robots operate in a 2 dimensional plane. In this project 6 degrees of freedom were offered and this was the first difficulty students experienced. The refraction of light in water also created difficulties using light sensors. Eventually the students learned to overcome these problems through the process of design and redesign.

After completing this semester-long project the students were very ecstatic and relieved that they overcame a challenging project. Their final presentations showed that their confidence in tackling a project was greatly increased and they were able to explain the principles and problems that hindered their design along the way.

Most groups required the entire duration of 12 weeks to complete the project, a few managed to finish early. Most students were happy to have done a very unique project that presented unexpected difficulties.

\section{Summary and Conclusion}

An underwater test field for underwater robots was constructed for under $\$ 1000$. Modifications to existing Lego Mindstorms equipment achieved waterproofing at minimal cost. Ten student teams that pioneered the first semester-long design project of AUVs were extremely enthusiastic. A more detailed assessment of this project will be done in the future. Student feedback will be used to enhance future versions of this project.

Bibliography

1. "Introductory Design in Freshman Engineering" Gunter W. Georgi and Lorcan M. Folan, 2001 
http://asee.org/acPapers/code/getPaper.cfm?paperID=3860\&pdf=00608_2001.PDF

2. "Introducing Design Throughout The Curriculum" Gunter W. Georgi, Lorcan M. Folan, and David R. Doucette, 2002

http://asee.org/acPapers/code/getPaper.cfm?paperID=4662\&pdf=2002-385_Final.pdf

3. "Project Management In Freshman Engineering" Onofrio N. Russo, Gunter W. Georgi, Lorcan M. Folan, 2003

http://asee.org/acPapers/2003-1901_Final.pdf

4. “A Simple Digital Logic Project For Freshman Engineering” David R. Doucette, Gunter W. Georgi, and Lorcan M. Folan, 2005

http://asee.org/acPapers/code/getPaper.cfm?paperID=8956\&pdf=2005-659_Final.pdf

5. "Building a LEGO ROV Using the MindStorms Robotics Kit" Amos G. Winter, 2001 http://www.mbari.org/education/internship/01interns/01papers/winter.pdf 